\title{
Novel germline mutations and unclassified variants of $B R C A 1$ and $B R C A 2$ genes in Chinese women with familial breast/ovarian cancer
}

Wen-Ming Cao ${ }^{1}$, Yun Gao ${ }^{2}$, Hong-Jian Yang ${ }^{3}$, Shang-Nao Xie ${ }^{3}$, Xiao-Wen Ding ${ }^{3}$, Zhi-Wen Pan ${ }^{4}$, Wei-Wu Ye and Xiao-Jia Wang ${ }^{1 *}$

\begin{abstract}
Background: Germline mutations in the BRCA1 and BRCA2 genes greatly increase a woman's risk of developing breast and/or ovarian cancer. The prevalence and distribution of such mutations differ across races/ethnicities. Several studies have investigated Chinese women with high-risk breast cancer, but the full spectrum of the mutations in these two genes remains unclear.
\end{abstract}

Methods: In this study, 133 unrelated Chinese women with familial breast/ovarian cancer living in Zhejiang, eastern China, were enrolled between the years 2008 and 2014. The complete coding regions and exon-intron boundaries of BRCA1 and BRCA2 were screened by PCR-sequencing assay. Haplotype analysis was performed to confirm BRCA1 and $B R C A 2$ founder mutations. In silico predictions were performed to identify the non-synonymous amino acid changes that were likely to disrupt the functions of BRCA1 and BRCA2.

Results: A total of 23 deleterious mutations were detected in the two genes in 31 familial breast/ovarian cancer patients with a total mutation frequency of $23.3 \%$ (31/133). The highest frequency of $50.0 \%(8 / 16)$ was found in breast cancer patients with a history of ovarian cancer. The frequencies of BRCA1 and BRCA2 mutations were $13.5 \%$ (18/133) and $9.8 \%$ (13/133), respectively. We identified five novel deleterious mutations (c.3295delC, c.3780_3781delAG, c.4063_4066delAATC, c.5161 > T and c.5173insA) in BRCA1 and seven (c.1-40delGA, c.4487delC, c.469_473delAAGTC, c.5495delC, c.6141T > A, c.6359C > G and c.7588C > T) in BRCA2, which accounted for $52.2 \%(12 / 23)$ of the total mutations. Six recurrent mutations were found, including four (c.3780_3781delAG, c.5154G > A, c.5468-1 del8 and c.5470_5477del8) in BRCA1 and two (c.3109C > T and c.5682C > G) in BRCA2. Two recurrent BRCA1 mutations (c.5154G > A and c.5468-1 del8) were identified as putative founder mutations. We also found 11 unclassified variants, and nine of these are novel. The possibility was that each of the non-synonymous amino acid changes would disrupt the function of BRCA1 and BRCA2 varied according to the different algorithms used.

Conclusions: BRCA1 and BRCA2 mutations accounted for a considerable proportion of hereditary breast/ovarian cancer patients from eastern China and the spectrum of the mutations of these two genes exhibited some unique features. The two BRCA1 putative founder mutations may provide a cost-effective option to screen Chinese population, while founder effects of the two mutations should be investigated in a lager sample size of patients.

Keywords: BRCA1, BRCA2, Germline mutation, Unclassified variants, Founder mutation, Chinese women

\footnotetext{
* Correspondence: wxiaojia@yahoo.com

'Department of Medical Oncology, Zhejiang Cancer Hospital, 38 Guangji

Road, Hangzhou 310022, China

Full list of author information is available at the end of the article
} 


\section{Background}

In 2009, the morbidity rate of breast cancer was 42.55 per 100,000 Chinese women, and breast cancer ranked first in cancer incidence and fifth in cancer-related deaths among females [1]. The mean age at diagnosis of breast cancer is 45-55 years in Chinese women, which is considerably younger than that in western women [2]. A significant proportion of breast cancer in Chinese women is caused by genetic alterations. Germline mutations in many genes, such as BRCA1, BRCA2, ATM, TP53, RAD51C and $X R C C 2$, have been identified to be associated with breast cancer [3-5]. Several studies have investigated germline mutations in genes including BRCA1, BRCA2, TP53, BRIP1, PALB2, CHEK2, RAD50, NBS1 and RAD51C in Chinese women with high risk breast cancer [6-21]. We previously summarized the spectrum of the germline mutations in these genes and found that the BRCA1 and $B R C A 2$ tumor suppressor genes are the two most important susceptibility genes and account for nearly $98 \%$ of hereditary breast cancer in China [22]. We found that the spectrum of BRCA1 and BRCA2 germline mutations in Chinese high risk breast cancer patients are much smaller than those in Caucasian patients, and little has been recognized in this field. The overall mutation frequencies in these two genes in Chinese high risk breast cancer patients ranged from 8.3 to $27.8 \%$, depending on the detection methods and patient inclusion criteria used. These frequencies are much lower than the $25-40 \%$ in BRCA1 and 6-15\% in BRCA2 that have been observed in Caucasian populations [22]. Because germline mutations in BRCA1 and $B R C A 2$ greatly increase a woman's risk of developing breast and/or ovarian cancer, and the prevalence and distribution of the germline mutations differ in different races/ ethnicities, we were interested in identifying the full spectrum of these mutations in high-risk female breast cancer patients in the Chinese population.

In this study, we screened the entire coding regions and exon-intron boundaries of the BRCA1 and BRCA2 genes in 133 familial breast/ovarian cancer patients from eastern China. A total of 23 deleterious mutations, including 12 novel mutations (five in $B R C A 1$ and seven in $B R C A 1$ ), were detected in these two genes in 31 familial breast/ovarian cancer patients, and the total mutation frequency was $23.3 \%(31 / 133)$. The highest frequency of $50.0 \%(8 / 16)$ was found in the breast cancer patients with a history of ovarian cancer. Six recurrent mutations were found, including four in BRCA1 and two in BRCA2. We also found 11 unclassified variants (UVs), nine of which were novel. Additionally, using comparative evolutionary bioinformatic programs, we identified the non-synonymous amino acid changes that are likely to disrupt the functions of the $B R C A 1$ and $B R C A 2$ genes. Our study suggested that $B R C A 1$ and BRCA2 mutations accounted for a considerable proportion of the hereditary breast/ovarian cancer patients in eastern China and that the spectrum of the mutations in these genes exhibited unique features.

\section{Methods \\ Subjects}

All patients were diagnosed between 2008 and 2014 in the Zhejiang Cancer Hospital, eastern China. The criterion for familial breast/ovarian cancer was that at least one first- or second-degree relative of the breast cancer patient had been affected by breast cancer and/or ovarian cancer, regardless of age. Written consent was obtained from all participating patients. The study was approved by the Research and Ethics Committee of Zhejiang Cancer Hospital, China. Peripheral blood samples were drawn from at least one affected person in each family and stored in EDTA tubes at $-80{ }^{\circ} \mathrm{C}$. A total of 133 patients from unrelated families were enrolled in this study. For the 62 patients who enrolled before 2012, the BRCA1 gene was analyzed with a polymerase chain reaction (PCR)-sequencing assay as previously reported [13], and the mutations of the BRCA2 gene were screened in this study.

\section{$B R C A 1$ and $B R C A 2$ mutation analysis}

Genomic DNA was extracted from the peripheral blood leukocytes of one patient from each family using a ZR Genomic DNA Kit (Zymo Research, Orange County, CA, USA) or a QIAamp DNA Blood Mini kit (Qiagen, Hilden, Germany). The entire coding regions and exon-intron boundaries of BRCA1 [U14680.1] and BRCA2 [U43746.1] were screened using PCR-sequencing assay. Totals of 32 pairs and 40 pairs of primers for BRCA1 and BRCA2, respectively, were synthesized by Invitrogen. The primers and PCR conditions are available on request. The PCR products were verified on standard agarose gels prior to mutation analysis and purified by membrane retention. The purified fragments were sequenced using a BigDye Terminator Cycle Sequencing Kit and an ABI 3130xl Genetic Analyzer (Applied Biosystems, Foster City, CA, USA). All mutations were confirmed by duplicate independent PCR. No screening for large genomic rearrangements was performed.

All of the mutations and variants were named according to the Human Genome Variation Sequence systematic nomenclature (HGVS; http://www.hgvs.org/mutnomen/). The Breast Cancer Information Core (BIC) nomenclature (https://research.nhgri.nih.gov/projects/bic/Member/index. shtml) was also indicated in the tables and text because this system had been widely employed in many studies. All of the mutations and variants were queried against the 1000 Genomes database using the 1000 Genomes Browser (http://browser.1000genomes.org/) to determine whether the mutations and variants had been reported in the Chinese population. 


\section{Haplotype analysis}

Haplotype analysis was conducted on the unrelated patients with recurrent $B R C A 1$ or $B R C A 2$ germline deleterious mutations. Thirteen microsatellite polymorphic loci were used (BRCA1 D17S855, D17S1322, D17S1323, D17S1326, D17S1327; BRCA2 D13S1304, D13S217, D13S289, D13S1699, D13S1698, D13S171, D13S1695, D13S267) [9, 12]. Primer sequences of all microsatellite polymorphic loci were obtained from the Probe Database (http://www.ncbi.nlm.nih.gov/probe). PCR products fluorescently labeled were size fractioned on an ABI 3730xl Analyzer (Applied Biosystems) using GeneScan 500 LIZ Size Standard. Analysis was performed using the Genemarker v1.5 analysis software.

\section{In silico prediction}

To identify the UVs that were likely to disrupt the functions of the BRCA1 and BRCA2 genes, we performed in silico predictions with the following six comparative evolutionary bioinformatic programs: Align-GVGD (http:// agvgd.iarc.fr/agvgd_input.php), SIFT (http://sift.jcvi.org/), PROVEAN (http://provean.jcvi.org/index.php), PolyPhen2 (http://genetics.bwh.harvard.edu/pph/), PMUT (http:// mmb2.pcb.ub.es:8080/PMut/), and PANTHER (http:// www.pantherdb.org/tools/csnpScoreForm.jsp).

\section{Statistical analysis}

Continuous data were presented as the mean \pm standard deviation (SD), and the differences between the two groups were evaluated using one-way ANOVA analyses. Frequencies were calculated as the proportion of mutation carriers among all participants. The differences in the overall frequencies of $B R C A 1$ and $B R C A 2$ mutations between groups were evaluated using Chi-square tests and Fisher's exact tests. The statistics were performed using SPSS version 17.0 software for Windows.

\section{Results \\ Patient features}

A total of 133 unrelated patients with personal and family histories of breast and/or ovarian cancer underwent BRCA1 and BRCA2 germline mutation screening. All of the patients were from the Zhejiang province in eastern China. In our cohort of 133 breast cancer families, there were 2.3 \pm 0.7 (mean number \pm SD) occurrences of breast cancer per family. The age of breast cancer onset ranged from 22 years to 74 years. The mean age at diagnosis was 43.0 \pm 9.3 (mean age $\pm \mathrm{SD}$ ) years. Ovarian cancer was present in $12.0 \%(16 / 133)$ of all families.

\section{BRCA1 deleterious mutations}

In this cohort of 133 familial breast/ovarian cancer patients, 13 deleterious mutations in BRCA1 were found in 18 unrelated patients, including five mutations that were reported in our previous study [13] (Table 1). None of the mutations had been registered in the 1000 Genomes database. The majority of the mutations were either nonsense or frameshift mutations with the exception of c.5467 +1G > A and c.5468-1del8. Six mutations (46.2\%) were located in exon 11 , and others were located in exon 19, exon 20, intron 23 and exon 24. There were five novel deleterious mutations (c.3295delC, c.3780_3781delAG, c.4063_4066delAATC, c.5161C > T and c.5173insA) that had not been registered in the $\mathrm{BIC}$ or any other public database. Moreover, two of the mutations (c.5468-1del8 and c.1465G > T) had only been previously reported in Chinese population. In this cohort, we detected four recurrent mutations (c.3780_3781delAG, c.5154G > A, c.5468-1del8 and c.5470_5477del8), which accounted for $30.8 \%(4 / 13)$ of the total mutations. The mutation c.5470_5477del8 occurred three times, and the others occurred twice. The mean age at diagnosis of these $B R C A 1$ mutation carriers was $39.9 \pm 8.1$ (mean age \pm SD) years (Table 2). No significant differences in the mean age at diagnosis between the BRCA1 mutation carriers, $B R C A 2$ mutation carriers and non-carriers were found.

\section{BRCA2 deleterious mutations}

A total of 10 deleterious mutations in $B R C A 2$ were found in 13 familial breast/ovarian cancer patients in this cohort (Table 1). None of these mutations had been registered in the 1000 Genomes database. The mean age at diagnosis of these BRCA2 mutation carriers was $41.1 \pm 6.5$ (mean age \pm SD) years (Table 2). Nine mutations were either nonsense or frameshift mutation, and the remaining mutation c.140delGA, which resulted in the deletion of a guanine in intron 1 and an adenine in exon 2, was a splicing site mutation. Sixty percent $(6 / 10)$ of the all of the mutations were located in exon 11. There were seven novel mutations (c.140delGA, c.4487delC, c. 469_473delAAGTC, c.5495delC, c.6141 T > A, c.6359C > G and c.7588C > T) in this cohort, and these mutations represented $70 \%(7 / 10)$ of the mutations in this gene. Two recurrent mutations (c.3109C $>\mathrm{T}$ and c.5682C $>\mathrm{G}$ ) were detected in this cohort, and both of them were registered in the BIC.

\section{Frequencies of $B R C A 1$ and $B R C A 2$ deleterious mutations}

A total of 23 deleterious mutations of BRCA1 and BRCA2 were identified in 31 familial breast/ovarian cancer patients, and the frequency was $23.3 \%$ (31/133; Table 3). The frequencies of $B R C A 1$ and $B R C A 2$ mutations were $13.5 \%$ $(18 / 133)$ and $9.8 \%(13 / 133)$, respectively.

In the subgroup analysis, the highest overall $B R C A 1$ and BRCA2 mutations rate was $50.0 \%(8 / 16)$ in the breast cancer patients with family histories of ovarian cancer. The overall mutation rate of the two genes in the patients who were diagnosed at or before the age of 40 was higher than that of the counterpart group. Compared with the breast cancer patients with fewer than two relatives affected by 
Table 1 BRCA1 and BRCA2 deleterious germline mutations in 133 Chinese women with familial breast/ovarian cancer

\begin{tabular}{|c|c|c|c|c|c|c|}
\hline Gene & No. of patient & Exon & Systematic nomenclature & BIC nomenclature & Amino acid change & References \\
\hline \multirow[t]{13}{*}{$B R C A 1$} & 1 & 11 & c. $1465 G>T$ & $1584 \mathrm{G}>\mathrm{T}$ & E489X & Zhi et al. [7] \\
\hline & 1 & 11 & c. $1945 G>T$ & $2064 G>T$ & E649X & $\mathrm{BIC}$ \\
\hline & 1 & 11 & c.3295delC & 3414delC & P1099LfsX10 & Novel \\
\hline & 2 & 11 & c.3780_3781delAG & 3899_3900delAG & L1260FfsX6 & Novel \\
\hline & 1 & 11 & c.4063_4066delAATC & 4182_4185delAATC & N1355KfsX10 & Novel \\
\hline & 1 & 11 & c.4065_4068delTCAA & 4184_4187delTCAA & N1355KfsX10 & $\mathrm{BIC}$ \\
\hline & 2 & 19 & C. $5154 G>A$ & $5273 G>A$ & W1718X & $\mathrm{BIC}$ \\
\hline & 1 & 19 & C.5161C > T & $5280 C>T$ & Q1721X & Novel \\
\hline & 1 & 19 & c.5173insA & 5292insA & E1725EfsX7 & Novel \\
\hline & 1 & 20 & c. $5251 C>T$ & $5370 \mathrm{C}>\mathrm{T}$ & R1751X & $\mathrm{BIC}$ \\
\hline & 1 & Intron23 & $c .5467+1 \mathrm{G}>\mathrm{A}$ & $\mathrm{IVS} 23+1 \mathrm{G}>\mathrm{A}$ & Splicing defect & $\mathrm{BIC}$ \\
\hline & 2 & Intron23 & c.5468-1del8 & 5587-1del8 & Splicing defect & Zhang et al. [11] \\
\hline & 3 & 24 & c.5470_5477del8 & 5589_5596del8 & I1824DfsX3 & $\mathrm{BIC}$ \\
\hline \multirow[t]{10}{*}{$B R C A 2$} & 1 & Intron1 & c.1-40delGA & IVS1-1deGA & Splicing defect & Novel \\
\hline & 1 & 5 & c.469_473delAAGTC & 697_701delAAGTC & K157SfsX24 & Novel \\
\hline & 1 & 9 & c.755_758delACAG & 983_986delACAG & T251XfsX1 & $\mathrm{BIC}$ \\
\hline & 2 & 11 & c.3109C > T & $3337 C>T$ & Q1037X & $\mathrm{BIC}$ \\
\hline & 1 & 11 & c.4487delC & 4715delC & P1496QfsX8 & Novel \\
\hline & 1 & 11 & c.5495delC & 5723delC & S1832LfsX8 & Novel \\
\hline & 3 & 11 & $c .5682 C>G$ & $5910 C>G$ & Y1894X & $\mathrm{BIC}$ \\
\hline & 1 & 11 & c.6141 T>A & $6369 \mathrm{~T}>\mathrm{A}$ & Y2047X & Novel \\
\hline & 1 & 11 & c. $6359 C>G$ & $6587 C>G$ & S2120X & Novel \\
\hline & 1 & 15 & c. $7588 \mathrm{C}>\mathrm{T}$ & $7816 \mathrm{C}>\mathrm{T}$ & Q2530X & Novel \\
\hline
\end{tabular}

BIC Breast Cancer Information Core

breast cancer or unilateral breast cancer, the overall mutation rates were higher in the patients with two or more relatives affected by breast cancer or bilateral breast cancer, but these differences did not reach statistical significance $(P=0.148$ and $P=0.115$, respectively).

\section{Haplotype analysis of recurrent mutations}

Four recurrent BRCA1 mutations (c.3780_3781delAG, c.5154G > A, c.5468-1del8 and c.5470_5477del8) and two recurrent BRCA2 mutations (c.3109C > T and c.5682C > G) were identified in unrelated breast cancer patients. As haplotype analysis of BRCA1 c.5470_5477del8 mutation and $B R C A 2$ c. $3109 \mathrm{C}>\mathrm{T}$ mutation had been performed in

Table 2 Mean age at diagnosis in different BRCA1 and BRCA2 status

\begin{tabular}{|c|c|c|c|c|c|c|}
\hline & $B R C A 1$ & $B R C A 2$ & Non-carriers & $p^{\mathrm{a}}$ & $p^{b}$ & $p^{c}$ \\
\hline Number & 18 & 13 & 102 & & & \\
\hline Mean age $( \pm S D)$ & $39.9( \pm 8.1)$ & $41.1( \pm 6.5)$ & $43.9( \pm 9.7)$ & 0.74 & 0.11 & 0.31 \\
\hline
\end{tabular}

Chinese high risk breast cancer patients [9, 10, 12], we performed haplotype analysis on the other four recurrent mutations in this study. Our results showed that carriers with the recurrent BRCA1 c.5154G > A mutation shared the same haplotype, as well as carriers with the recurrent BRCA1 c.5468-1del8 mutation, which suggested that these two putative founder mutations were derived from a common ancestor (Table 4). The three carriers with BRCA2 c.5682C > G mutation sharing only two alleles (D13S171 and D13S1698) out of eight alleles implied that they might be not derived from a common ancestor (Table 5).

\section{UVs of $B R C A 1$ and $B R C A 2$}

In addition to deleterious mutations, we identified $11 \mathrm{UVs}$ (seven in BRCA1 and four in BRCA2; Table 6). Comparisons with the 1000 Genomes database revealed that only BRCA1 c. $2286 \mathrm{~A}>\mathrm{T}$ (R762S) had been reported in a Pakistani population, and the frequency of the $\mathrm{T}$ allele was $0.5 \%$ in that population. None of the UVs had previously been found in the Chinese population. The majority of the variants were novel, with the exception of the mutation c. $2286 \mathrm{~A}>\mathrm{T}$ in $\mathrm{BRCA1}$, which is registered in the $\mathrm{BIC}$, and c. $2726 \mathrm{~A}>\mathrm{T}$ in $B R C A 1$, which was recently reported in a 
Table 3 Frequencies of BRCA1 and BRCA2 germline deleterious mutations in different groups of patients

\begin{tabular}{|c|c|c|c|c|c|}
\hline Features & Number of total cases & BRCA1 mutation (\%) & BRCA2 mutation (\%) & Overall mutation (\%) & $P$-value \\
\hline Total & 133 & $18(13.5)$ & $13(9.8)$ & $31(23.3)$ & \\
\hline \multicolumn{6}{|l|}{ Age at onset } \\
\hline$\leq 40$ years & 51 & $11(21.6)$ & $6(11.8)$ & $17(33.3)$ & \multirow[t]{2}{*}{0.031} \\
\hline$>40$ years & 82 & $7(8.5)$ & $7(8.5)$ & $14(17.1)$ & \\
\hline \multicolumn{6}{|c|}{ Number of breast cancer cases in a family } \\
\hline$\leq 2$ & 99 & $12(12.1)$ & $8(8.1)$ & $20(20.2)$ & \multirow[t]{2}{*}{0.148} \\
\hline$>2$ & 34 & $6(17.6)$ & $5(14.7)$ & $11(32.4)$ & \\
\hline \multicolumn{6}{|c|}{ With a family history of ovarian cancer } \\
\hline Yes & 16 & $6(37.5)$ & $2(12.5)$ & $8(50.0)$ & \multirow[t]{2}{*}{0.012} \\
\hline No & 117 & $12(10.3)$ & $11(9.4)$ & $23(19.7)$ & \\
\hline \multicolumn{6}{|c|}{ Bilateral breast cancer } \\
\hline Yes & 15 & $3(20)$ & $3(20)$ & $6(40)$ & \multirow[t]{2}{*}{0.115} \\
\hline No & 118 & 15 (12.7) & $10(8.5)$ & $25(21.2)$ & \\
\hline
\end{tabular}

Chinese population previously [8]. The possibility that each of the UVs would disrupt the function of BRCA1 or $B R C A 2$ was predicted in silico, and the results varied according to the different algorithms used.

\section{Discussion}

$B R C A 1$ and $B R C A 2$ are the most important genetic susceptibility genes for breast/ovarian cancer in both Caucasian and Chinese populations. The spectrum and frequencies of mutations in these two genes in Chinese women with familial breast/ovarian cancer have been insufficiently explored to date. Moreover, the penetrance has not yet been investigated. Due to the limited knowledge on hereditary breast/ovarian cancer, there is no genetic counseling or testing services available in Mainland China.

Our results demonstrated that the frequency of $B R C A 1$ and $B R C A 2$ mutations among Chinese women with familial breast/ovarian cancer was $23.3 \%$. Similar results have been reported in the Korean population [23], Hispanic population [24] and Africa American population [25]. However, the frequency observed in the current study is lower than that reported in an Ashkenazi Jewish population, in which the frequency of BRCA1 and BRCA2 mutations was $69 \%$ [25]. Compared with other reports about Chinese populations, the frequency found in our cohort was the highest in patients with familial breast/ ovarian cancer. Li et al. [9] used PCR-DHPLC assay to screen for BRCA1 and BRCA2 mutations in 241 women with familial breast cancer from northern or southern China and found a frequency of $12.9 \%$. Although the PCR-DHPLC assay is cost-effective for screening for genetic mutations, a considerable number of diseaseassociated mutations may have been missed by this indirect detection method [26]. Zhang et al. [11] reported that the frequency of BRCA1 and BRCA2 mutations in northern Chinese familial breast cancer patients was $10.5 \%$ (43/409) based on PCR-sequencing assay. The enrolment criteria and mutation detecting assay used in this were comparable with the criteria used in our study, but the reported frequency was much lower than that observed in the present study. In their subgroup analysis, the highest frequency was $23 \%$ in the patients whose tumors had been diagnosed at or before the age of 40 years. However, the frequency reached $33.3 \%$ in this group of patients in our cohort. Moreover, in the study conducted by Kwong et al., [12] the frequency of $B R C A 1$ and $B R C A 2$ mutations in high-risk breast/ovarian cancer patients was $15.3 \%$ (69/

Table 4 Haplotype analysis of BRCA1 recurrent mutations carriers

\begin{tabular}{|c|c|c|c|c|c|c|}
\hline Mutation & Patient No. & D17S855 & D17S1322 & D17S1323 & D17S1326 & D17S1327 \\
\hline \multirow[t]{2}{*}{ c.3780_3781delAG } & 1 & $145 / 147$ & $113 / 116$ & $150 / 152$ & $108 / 110$ & $128 / 130$ \\
\hline & 2 & $141 / 143$ & 119/122 & $156 / 160$ & $86 / 88$ & $158 / 160$ \\
\hline \multirow[t]{2}{*}{ c. $5154 \mathrm{G}>\mathrm{A}$} & 3 & $143 / 141$ & $122 / 119$ & $156 / 156$ & $90 / 88$ & $154 / 152$ \\
\hline & 4 & $143 / 151$ & $122 / 116$ & $156 / 152$ & $90 / 104$ & $154 / 130$ \\
\hline \multirow[t]{2}{*}{ c.5468-1 del8 } & 5 & $147 / 141$ & $116 / 122$ & $152 / 146$ & $104 / 106$ & $128 / 130$ \\
\hline & 6 & $147 / 145$ & $116 / 113$ & $152 / 150$ & $104 / 102$ & $128 / 130$ \\
\hline
\end{tabular}


Table 5 Haplotype analysis of BRCA2 C.5682C > G mutation carriers

\begin{tabular}{lllllllll}
\hline Patient No. & D13S171 & D13S217 & D13S267 & D13S289 & D13S1304 & D13S1695 & D13S1698 & D13S1699 \\
\hline 7 & $\mathbf{2 2 4} / 238$ & $\mathbf{1 6 4 / 1 7 2}$ & $\mathbf{1 4 4 / 1 5 1}$ & $\mathbf{1 4 6 / 1 5 6}$ & $157 / 159$ & $\mathbf{2 1 1 / 2 1 5}$ & $\mathbf{1 5 8 / 1 5 6}$ & $161 / 163$ \\
8 & $\mathbf{2 2 4 / 2 2 4}$ & $168 / 170$ & $151 / 159$ & $144 / 156$ & $\mathbf{1 5 3 / 1 5 5}$ & $\mathbf{2 0 9 / 2 1 3}$ & $\mathbf{1 5 8 / 1 5 6}$ & $\mathbf{1 5 7 / 1 5 5}$ \\
9 & $\mathbf{2 2 4 / 2 2 8}$ & $\mathbf{1 6 4 / 1 6 0}$ & $\mathbf{1 4 4 / 1 4 2}$ & $\mathbf{1 4 6 / 1 4 4}$ & $\mathbf{1 5 3 / 1 4 9}$ & $\mathbf{2 1 1 / 2 0 7}$ & $\mathbf{1 5 8 / 1 6 0}$ & $\mathbf{1 5 7 / 1 5 9}$ \\
\hline
\end{tabular}

Shared haplotypes are bolded

651). These authors also employed the conventional PCRsequencing assay, and the patients were recruited from southern China. The proportion of high-risk breast/ovarian cancer patients, including familial breast cancer patients and early-onset cases and the frequency of two-gene mutations were much lower in the early-onset patients than in the familial breast cancer cases. Large genomic rearrangements account for 4-28\% of all BRCA1 and BRCA2 mutations [27], and such mutations have been found in Chinese women at a high risk for breast cancer [28-32]. Because the PCR-sequencing assay cannot detect these rearrangements, the frequency of mutations in our cohort might have been underestimated, and the frequency of $B R C A 1$ and $B R C A 2$ mutations in the eastern Chinese population could be significant.

Although several studies have reported that the $B R C A 2$ mutations are more frequent than $B R C A 1$ mutations in Asian population [11, 12, 33, 34], BRCA1 mutations seemed to be more prevalent in our cohort. This finding might be attributable to two points. First, most studies have reported that BRCA2 mutations predominantly occur in relatively late-onset breast cancer patients compared with BRCA1 mutations [11,35], but the patients enrolled in our study were much younger than those in other studies, which might have resulted in an underestimation of the contribution of BRCA2 mutations. Second, a greater number of recurrent mutations were found in BRCA1 than in $B R C A 2$ in our study, which elevated the frequency of BRCA1 mutations.

In the present study, we found that $52.2 \%(12 / 23)$ of the deleterious mutations were novel; these mutations included five mutations in BRCA1 and seven mutations in BRCA2. In our previous systemic analysis of the spectrum of BRCA1 and BRCA2 mutations in Han Chinese women, we reported that $56.3 \%(40 / 71)$ and $47.9 \%(35 / 73)$ of the $B R C A 1$ and $B R C A 2$ mutations were novel, respectively [22]. It seems that the spectrum of BRCA1 and BRCA2 mutations in Chinese women exhibit unique features. The BRCA2 mutation c.1-40delGA in our cohort was novel. Bakker et al. [36] found a BRCA2 c.1-40 G > A mutation in a Japanese Fanconi anemia family. The functional analysis of these authors used a mouse embryonic stem cell-based assay that revealed that this mutation caused aberrant splicing, reduced transcript levels and hypersensitivity to DNA damaging agents, suggesting that this mutation was likely pathogenic. These authors thought that this finding was relevant for mutation analysis in hereditary breast and ovarian cancer syndrome families in a diagnostic setting. The mutation c.1-40delGA, which deletes a guanine in intron 1 and an adenine in exon 2 and causes the loss of the donor site of intron 1 , should also be pathogenic.

Six BRCA1 and BRCA2 recurrent mutations were identified in multiple patients, and these accounted for $45.2 \%$ $(14 / 31)$ of the total patients with mutations. Of these mutations, one (c.3780_3781delAG) was novel, another (c.5468-1del8) was recently reported in Chinese women [11], and the remaining four had been reported in the BIC database. Founder mutations provide population-specific genetic risk assessment, and facilitate genetic mutation screening. Thus far, few studies have suggested that putative founder mutations of BRCA1 and BRCA2 might exist in Chinese women at a high risk for breast cancer, such as the c.981delAT and c.5470_5477del8 mutations in BRCA1 and the c.3109C > T, c.7436_7805del370 and c.9097_9098insA mutations in BRCA2 [9, 10, 12]. In our cohort, the BRCA1 c.5470_5477del8 mutation and BRCA2 c.3109C $>$ T mutation were both recurrent, but no other three putative founder mutations was found. Our haplotype analysis revealed that BRCA1 c.5154G > A and c.5468-1del8 mutations were the two putative founder mutations. Since there are only two patients reported for each of the putative founder mutation, the founder effects are needed to be investigated by larger sample size of patients. In our previous study, we reported that the most common recurrent mutations in Chinese women at high risk for breast cancer are c.5470_5477del8 in BRCA1 and c.3109C $>\mathrm{T}$ in BRCA2 [22], which were reported to be the putative founder mutations. However, the study that enrolled the greatest number of familial breast cancer patients from northern China did not find these six putative founder mutations except the BRCA1 c.5468-1del8 mutation [11]. The discrepancy regarding the founder mutations in Chinese familial breast cancer patients may be due to geographic differences. The characterization of BRCA1 and BRCA2 founder mutations and association between the founder mutations and breast cancer risk should be studied in a large-scale Chinese population size.

Although, elevated mutation rates of $B R C A 1$ and $B R C A 2$ were found in patients who had been diagnosed at or before 40 years of age, no significant differences were found between the BRCA1 mutation carriers, BRCA2 mutation carriers and non-carriers when compared to a mean age at 
Table 6 BRCA1 and BRCA2 germline UVs in 133 Chinese women with familial breast/ovarian cancer

\begin{tabular}{|c|c|c|c|c|c|c|c|c|c|c|c|c|}
\hline Gene & $\begin{array}{l}\text { No. of } \\
\text { patient }\end{array}$ & Exon & $\begin{array}{l}\text { Systematic } \\
\text { nomenclature }\end{array}$ & $\begin{array}{l}\text { BIC } \\
\text { nomenclature }\end{array}$ & $\begin{array}{l}\text { Amino acid } \\
\text { change }\end{array}$ & References & $\begin{array}{l}\text { Align- } \\
\text { GVGD }\end{array}$ & SIFT & PROVEAN & PolyPhen-2 & PMUT & PANTHER \\
\hline \multirow[t]{7}{*}{$\overline{B R C A 1}$} & 1 & 11 & c.1679A > T & $1798 \mathrm{~A}>\mathrm{T}$ & D560V & Novel & $\mathrm{CO}$ & Damaging & Deleterious & $\begin{array}{l}\text { Possibly } \\
\text { damaging }\end{array}$ & Neutral & $\overline{\text { Deleterious }}$ \\
\hline & 1 & 11 & c. $1537 C>G$ & $1656 C>G$ & H513D & novel & $\mathrm{CO}$ & Tolerated & Deleterious & Benign & Pathological & Neutral \\
\hline & 1 & 11 & $c .2286 \mathrm{~A}>\mathrm{T}$ & $2405 \mathrm{~A}>\mathrm{T}$ & R762S & $\mathrm{BIC}$ & CO & Damaging & Deleterious & Benign & Pathological & Neutral \\
\hline & 1 & 14 & c. $4445 \mathrm{~A}>C$ & $4564 A>C$ & D1482A & novel & CO & Damaging & Neutral & Benign & Pathological & Neutral \\
\hline & 1 & 11 & c.1966A $>$ T & $2085 A>T$ & N656Y & novel & CO & Damaging & Deleterious & $\begin{array}{l}\text { Possibly } \\
\text { damaging }\end{array}$ & Neutral & Deleterious \\
\hline & 1 & 11 & $c .2340 G>T$ & $2459 \mathrm{G}>\mathrm{T}$ & $\mathrm{Q} 780 \mathrm{H}$ & novel & CO & Damaging & Deleterious & $\begin{array}{l}\text { Probably } \\
\text { damaging }\end{array}$ & Neutral & Deleterious \\
\hline & 1 & 11 & $c .2726 \mathrm{~A}>\mathrm{T}$ & $2845 \mathrm{~A}>\mathrm{T}$ & N909| & $\begin{array}{l}\text { BIC, Thirthagiri et al. } \\
\text { [8] }\end{array}$ & $\mathrm{CO}$ & Damaging & Deleterious & $\begin{array}{l}\text { Possibly } \\
\text { damaging }\end{array}$ & Neutral & Neutral \\
\hline \multirow[t]{4}{*}{ BRCA2 } & 1 & 10 & c.1568A > G & $1796 \mathrm{~A}>\mathrm{G}$ & $\mathrm{H} 523 \mathrm{R}$ & novel & $\mathrm{CO}$ & Damaging & Neutral & Benign & Pathological & Neutral \\
\hline & 1 & 11 & c. $3904 A>G$ & $4132 A>G$ & T1302A & novel & CO & Tolerated & Deleterious & Benign & Neutral & Neutral \\
\hline & 1 & 11 & c. $5590 \mathrm{G}>\mathrm{A}$ & $5818 \mathrm{G}>\mathrm{A}$ & D1864N & novel & CO & Damaging & Neutral & Benign & Neutral & Neutral \\
\hline & 1 & 11 & c. $6763 \mathrm{~A}>\mathrm{T}$ & $6991 \mathrm{~A}>\mathrm{T}$ & T2255S & novel & $\mathrm{CO}$ & Damaging & Neutral & $\begin{array}{l}\text { Possibly } \\
\text { damaging }\end{array}$ & Neutral & Deleterious \\
\hline
\end{tabular}


diagnosis. The inconsistent results implied that these observations did not withstand multiple comparisons in our cohort. Breast cancer patients with family histories of ovarian cancer exhibited the highest overall mutation rate of BRCA1 and BRCA2, which implied that BRCA1 and $B R C A 2$ mutations are more likely to occur in families with a history of both breast and ovarian cancer. This result is consistent with those of other studies $[9,11]$.

Eleven UVs were found in our study, and the potentials for these variants to disrupt the functions of BRCA1 and $B R C A 2$ varied according to the algorithm program used. The UVs accounted for nearly $1 / 3$ of the total mutations/ variants in this study. The risks of breast and ovarian cancer in the UVs carriers might be as high as those in the carriers of the classical pathogenic mutations. A variety of approaches have been used to investigate the clinical relevance of these UVs. Co-segregation analysis is regarded as a robust approach because it is directly related to the disease risk and is not affected by selection bias [37]. The absence of co-segregation provides strong evidence against pathogenicity. Unfortunately, the samples required for us to perform co-segregation analysis of UVs and the deleterious mutations in the multi-tumor families were not available.

\section{Conclusions}

In the present study, we found that the frequency of $B R C A 1$ and BRCA2 mutations was $23.3 \%$ in our cohort of 133 Chinese women with familial breast/ovarian cancer, and the frequency of BRCA1 and BRCA2 mutations was $50 \%$ in patients with a familial history of both breast cancer and ovarian cancer. The spectrum of BRCA1 and $B R C A 2$ mutations in the Chinese population are quite different from those in other ethnicities. Six recurrent mutations were detected in this study, in which two recurrent $B R C A 1$ mutations were identified as putative founder mutations, and a larger sample size is required to determine the founder effects of these two mutations in Chinese women. BRCA1 and BRCA2 mutations account for a considerable proportion of Chinese hereditary breast/ovarian cancer patients, and the penetrance of these two genes should be investigated because such investigations will be very important for the development of a preventive treatment strategy in China.

\section{Abbreviations}

BIC: Breast cancer information core; PCR: Polymerase chain reaction; SD: Standard deviation; UVs: Unclassified variants.

\section{Competing interests}

The authors declare that they have no competing interests.

\section{Authors' contributions}

WMC: designed the study, analyzed the mutational data, performed haplotype analysis and drafted the manuscript. YG and ZWP: performed PCR and sequencing studies. HJY, SNX and XWD: collected the clinical and pathological data. WWY: performed the statistical analysis. XJW: conceived of the study, participated in its design and coordination and helped to draft the manuscript. All authors read and approved the final manuscript.

\section{Acknowledgements}

This research was supported by the grants from Science and Technology Program offered by Health Bureau of Zhejiang Province, China (Grant numbers: 2007A023, 2012RCB006 and 2014KYA006) and Zhejiang Province Traditional Medical Science Fund Project of China (Grant number: 2012ZB019).

\section{Author details}

'Department of Medical Oncology, Zhejiang Cancer Hospital, 38 Guangji Road, Hangzhou 310022, China. ${ }^{2}$ Institute of Cancer Research, Zhejiang Cancer Hospital, Hangzhou 310022, China. ${ }^{3}$ Department of Breast Cancer Surgery, Zhejiang Cancer Hospital, Hangzhou 310022, China. ${ }^{4}$ Department of Clinical Laboratory, Zhejiang Cancer Hospital, Hangzhou 310022, China.

Received: 7 December 2014 Accepted: 1 February 2016

Published online: 06 February 2016

\section{References}

1. Chen W, Zheng R, Zhang S, Zhao P, Li G, Wu L, et al. Report of incidence and mortality in China cancer registries. Chin J Cancer. 2013;32:106-12

2. Fan L, Strasser-Weippl K, Li JJ, St Louis J, Finkelstein DM, Yu KD, et al. Breast cancer in China. Lancet Oncol. 2014;15:e279-289.

3. Ripperger T, Gadzicki D, Meindl A, Schlegelberger B. Breast cancer susceptibility: current knowledge and implications for genetic counselling. Eur J Hum Genet. 2009:17:722-31.

4. Meindl A, Hellebrand H, Wiek C, Erven V, Wappenschmidt B, Niederacher D, et al. Germline mutations in breast and ovarian cancer pedigrees establish RAD51C as a human cancer susceptibility gene. Nat Genet. 2010;42:410-4.

5. Giles GG, Devilee P, Benitez J, Hopper JL, Tavtigian SV, Goldgar DE, et al. Rare mutations in XRCC2 increase the risk of breast cancer. Am J Hum Genet. 2012; 90:734-9.

6. Li SS, Tseng HM, Yang TP, Liu CH, Teng SJ, Huang HW, et al. Molecular charaterization of germline mutations in BRCA1 and BRCA2 genes form breast cancer families in Taiwan. Hum Genet. 1999;104:201-4.

7. Zhi X, Szabo C, Chopin S, Suter N, Wang QS, Ostrander EA, et al. BRCA1 and BRCA2 sequence variants in Chinese breast cancer families. Hum Mutat. 2002;20:474

8. Thirthagiri E, Lee SY, Kang P, Lee DS, Toh GT, Selamat S, et al. Evaluation of BRCA1 and BRCA2 mutations and risk-prediction models in a typical Asian county (Malaysia) with a relatively low incidence of breast cancer. Breast Cancer Res. 2008;10:R59.

9. Li WF, Hu Z, Rao NY, Song CG, Zhang B, Cao MZ, et al. The prevalence of BRCA1 and BRCA2 germline mutations in high-risk breast cancer patients of Chinese Han nationality: two recurrent mutations were identified. Breast Cancer Res Treat. 2008:110:99-109.

10. Kwong A, Wong LP, Wong HN, Law FB, Ng EK, Tang YH, et al. A BRCA2 founder mutation and seven novel deleterious BRCA mutations in southern Chinese women with breast and ovarian cancer. Breast Cancer Res Treat. 2009;117:683-6.

11. Zhang J, Pei R, Pang Z, Ouyang T, Li J, Wang T, et al. Prevalence and characterization of BRCA1 and BRCA2 germline mutations in Chinese women with familial breast cancer. Breast Cancer Res Treat. 2012;132:421-8.

12. Kwong A, Ng EK, Wong CL, Law FB, Au T, Wong HN, et al. Identification of BRCA1/2 founder mutations in Southern Chinese breast cancer patients using gene sequencing and high resolution DNA melting analysis. PLoS One. 2012;7:e43994

13. Cao W, Wang X, Gao Y, Yang H, Li JC. BRCA1 Germ-line mutations and tumor characteristics in eastern Chinese women with familial breast cancer. Anat Rec (Hoboken). 2013;296:273-8

14. Cao AY, Jin W, Shi PC, Di GH, Shen ZZ, Shao ZM. Identification and characterization of two novel germ line p53 mutations in the non-LFS/non-LFL breast cancer families in Chinese population. Breast Cancer Res Treat. 2010;119: 295-303.

15. Cao AY, Huang J, Hu Z, Li WF, Ma ZL, Tang LL, et al. Mutation analysis of BRIP1/BACH1 in BRCA1/BRCA2 negative Chinese women with early onset breast cancer or affected relatives. Breast Cancer Res Treat. 2009;115:51-5.

16. Cao AY, Huang J, Hu Z, Li WF, Ma ZL, Tang LL, et al. The prevalence of PALB2 germline mutations in BRCA1/BRCA2 negative Chinese women with early onset breast cancer or affected relatives. Breast Cancer Res Treat. 2009; 114:457-62. 
17. Chen W, Yurong S, Liansheng N. Breast cancer low-penetrance allele 1100delC in the CHEK2 gene: not present in the Chinese familial breast cancer population. Adv Ther. 2008;25:496-501.

18. Thirthagiri E, Cheong LS, Yip CH, Teo SH. CHEK2*1100delC does not contribute to risk to breast cancer among Malay, Chinese and Indians in Malaysia. Fam Cancer. 2009;8:355-8.

19. Liu Y, Liao J, Xu Y, Chen W, Liu D, Ouyang T, et al. A recurrent CHEK2 p.H371Y mutation is associated with breast cancer risk in Chinese women. Hum Mutat. 2011;32:999-1003.

20. He M, Di GH, Cao AY, Hu Z, Jin W, Shen ZZ, et al. RAD50 and NBS1 are not likely to be susceptibility genes in Chinese non-BRCA1/2 hereditary breast cancer. Breast Cancer Res Treat. 2012;133:111-6.

21. Pang Z, Yan L, Zhang J, Ouyang T, Li J, Wang T, et al. RAD51C germline mutations in Chinese women with familial breast cancer. Breast Cancer Res Treat. 2011:129:1019-20.

22. Cao W, Wang X, Li JC. Hereditary breast cancer in the Han Chinese population. J Epidemiol. 2013;23:75-84.

23. Han SA, Kim SW, Kang E, Park SK, Ahn SH, Lee MH, et al. The prevalence of BRCA mutations among familial breast cancer patients in Korea: results of the Korean Hereditary Breast Cancer study. Fam Cancer. 2013;12:75-81.

24. Weitzel JN, Clague J, Martir-Negron A, Ogaz R, Herzog J, Ricker C, et al. Prevalence and type of BRCA mutations in Hispanics undergoing genetic cancer risk assessment in the southwestern United States: a report from the Clinical Cancer Genetics Community Research Network. J Clin Oncol. 2013;31:210-6.

25. Nanda R, Schumm LP, Cummings S, Fackenthal JD, Sveen L, Ademuyiwa F, et al. Genetic testing in an ethnically diverse cohort of high-risk women: a comparative analysis of BRCA1 and BRCA2 mutations in American families of European and African ancestry. JAMA. 2005;294:1925-233.

26. Klein $B$, Weirich $\mathrm{G}$, Brauch $\mathrm{H}$. DHPLC-based germline mutation screening in the analysis of the VHL tumor suppressor gene: usefulness and limitations. Hum Genet. 2001;108:376-84.

27. Mazoyer S. Genomic rearrangements in the BRCA1 and BRCA2. Hum Mutat. 2005:25:415-22.

28. Kwong A, Ng EK, Law FB, Wong HN, Wa A, Wong CL, et al. MA ES: Novel BRCA1 and BRCA2 genomic rearrangements in Southern Chinese breast/ ovarian cancer patients. Breast Cancer Res Treat. 2012;136:931-3.

29. Yap KP, Ang P, Lim IH, Ho GH, Lee AS. Detection of a novel Alu-mediated BRCA1 exon 13 duplication in Chinese breast cancer patients and implications for genetic testing. Clin Genet. 2006;70:80-2.

30. Lim YK, Lau PT, Ali AB, Lee SC, Wong JE, Putti TC, et al. Identification of novel BRCA large genomic rearrangements in Singapore Asian breast and ovarian patients with cancer. Clin Genet. 2007;71:331-42.

31. Kwong A, Ng EK, Tang EY, Wong CL, Law FB, Leung CP, et al. A novel de novo BRCA1 mutation in a Chinese woman with early onset breast cancer. Fam Cancer. 2011;10:233-7.

32. Kang P, Mariapun S, Phuah SY, Lim LS, Liu J, Yoon SY, et al. Large BRCA1 and BRCA2 genomic rearrangements in Malaysian high risk breast-ovarian cancer families. Breast Cancer Res Treat. 2010;124:579-84.

33. Kang E, Seong MW, Park SK, Lee JW, Lee J, Kim LS, et al. Korean Hereditary Breast Cancer Study Group: The prevalence and spectrum of BRCA1 and BRCA2 mutations in Korean population: recent update of the Korean Hereditary Breast Cancer (KOHBRA) study. Breast Cancer Res Treat. 2015;151:157-168.

34. Kim H, Choi DH. Distribution of BRCA1 and BRCA2 mutations in Asian patients with breast cancer. J Breast Cancer. 2013;16:357-65.

35. El Saghir NS, Zgheib NK, Assi HA, Khoury KE, Bidet Y, Jaber SM, et al. BRCA1 and BRCA2 mutations in ethnic Lebanese Arab women with high hereditary risk breast cancer. Oncologist. 2015;20:357-64.

36. Bakker JL, Thirthagiri $E$, van Mil SE, Adank MA, Ikeda H, Verheul HM, et al. A novel splice site mutation in the noncoding region of BRCA2: implications for Fanconi anemia and familial breast cancer diagnostics. Hum Mutat. 2014;35:442-6

37. Mohammadi L, Vreeswijk MP, Oldenburg R, van den Ouweland A, Oosterwijk JC, van der Hout AH, et al. A simple method for co-segregation analysis to evaluate the pathogenicity of unclassified variants; BRCA1 and BRCA2 as an example. BMC Cancer. 2009:9:211.

\section{Submit your next manuscript to BioMed Central and we will help you at every step:}

- We accept pre-submission inquiries

- Our selector tool helps you to find the most relevant journal

- We provide round the clock customer support

- Convenient online submission

- Thorough peer review

- Inclusion in PubMed and all major indexing services

- Maximum visibility for your research

Submit your manuscript at www.biomedcentral.com/submit

) Biomed Central 